\title{
Exploring the role of attention during memory retrieval: Effects of semantic encoding and divided attention
}

\author{
JEFFREY P. LOZITO and NEIL W. MULLIGAN \\ University of North Carolina, Chapel Hill, North Carolina
}

\begin{abstract}
Research on attention and memory suggests that semantic encoding leads to retrieval that is highly susceptible to divided attention. Three experiments tested this proposition and showed that dividing attention did not selectively affect semantically encoded items. Participants encoded a list of words in one of two ways: semantically or phonetically. Later, memory was assessed using either a standard recognition test (Experiment 1) or a rhyme recognition test (Experiments 2 and 3). The participants took the memory test either alone (full attention) or while simultaneously performing a secondary task (divided attention). Recognition accuracy was reduced by divided attention on both recognition tests, and semantically and phonetically encoded words were equally affected.
\end{abstract}

The traditional view of memory and attention states that attention is critical for the formation of durable memory traces (e.g., Broadbent, 1958; Cherry, 1953; James, 1890; Norman, 1969). Consistent with this view are the numerous studies showing that dividing attention during memory encoding reduces later memory performance (e.g., Baddeley, Lewis, Eldridge, \& Thomson, 1984; Craik, Govoni, Naveh-Benjamin, \& Anderson, 1996; Mulligan, 1998; Murdock, 1965). Because the traditional view posits a critical role for attention in memory encoding, it is not surprising that the bulk of research on attention and memory has focused on encoding, rather than on retrieval.

With the exception of a few early studies (e.g., Johnston, Greenberg, Fisher, \& Martin, 1970; Martin, 1970; Trumbo \& Milone, 1971), examining the effects of divided attention (DA) during memory retrieval is a more recent development in the literature. Baddeley et al. (1984) used a variety of secondary tasks (e.g., card sorting and digit load) to examine the effects of DA on both memory encoding and retrieval, using free recall, cued recall, and recognition memory tests. Dividing attention during encoding consistently disrupted performance on later memory tests. In contrast, dividing attention during retrieval produced little or no decrease in memory accuracy, leading Baddeley et al. to conclude that retrieval processes are relatively automatic.

Craik et al. (1996) came to a somewhat different conclusion regarding the automaticity of retrieval but agreed that dividing attention during retrieval produced minimal effects on memory accuracy. In this experiment, partici-

Correspondence concerning this article should be addressed to J. P. Lozito or N. W. Mulligan, Department of Psychology, University of North Carolina, Chapel Hill, NC 27599-3270 (e-mail: jlozito@email .unc.edu or nmulligan@unc.edu). pants retrieved and encoded words either in a full attention (FA) or a DA condition. In the DA condition, the secondary task was a continuous response task (CRT). Craik et al. assessed the role of attention in memory retrieval (and encoding) in two ways. First, accuracy in the memory task was compared under the FA and DA conditions (as in Baddeley et al.'s, 1984, study). Second, performance on the CRT task (the secondary task) in the DA condition was compared with performance on this task when it was performed alone (in a baseline condition, in which the CRT task was the sole task). This determines whether retrieval (or encoding) disrupts performance on the accompanying secondary task. Such secondary task costs are a traditional measure of attention costs.

The results indicated that dividing attention during memory retrieval produced little decline in accuracy (especially for recognition tests), replicating the results in Baddeley et al. (1984). However, reaction times (RTs) on the CRT task were longer when this task was paired with memory retrieval (or encoding, although secondary task costs were greater for retrieval than for encoding; Craik et al., 1996; see Naveh-Benjamin, Craik, Guez, \& Dori, 1998, for similar results). This replicated the findings of earlier studies (Johnston et al., 1970; Martin, 1970; Trumbo \& Milone, 1971) and contradicts the proposal of Baddeley et al. that retrieval processes are wholly automatic. Craik et al. concluded that unlike encoding processes, which are susceptible to DA effects, retrieval processes are obligatory (or protected) and, thus, not hindered by dividing attention (Craik et al., 1996; Naveh-Benjamin, Craik, Perretta, \& Tonev, 2000). Under this view, retrieval is argued to take precedence over other ongoing activities, protecting retrieval accuracy from the deleterious effects of a secondary task.

Despite these differences, both Baddeley et al. (1984) and Craik et al. (1996) argued that the key finding is that 
DA produces different effects on encoding and retrieval, disrupting memory accuracy in the former, but not in the latter. Craik, Naveh-Benjamin, and their colleagues have reported this result a number of times (Anderson, Craik, \& Naveh-Benjamin, 1998; Craik et al., 1996; NavehBenjamin \& Guez, 2000; Naveh-Benjamin, Craik, Gavrilescu, \& Anderson, 2000; Naveh-Benjamin et al., 1998; Naveh-Benjamin, Craik, Perretta, \& Tonev, 2000). The dissociative effects of attention on encoding and retrieval are most marked in recognition memory. That is, under the same experimental conditions, DA sometimes produces significant effects on free and cued recall without producing a comparable effect on recognition accuracy (e.g., Anderson et al., 1998; Park, Smith, Dudley, \& Lafronza, 1989). Consequently, we will focus on DA and recognition memory.

Despite the large number of studies reporting no DA effect on retrieval - and recognition memory, in particulardivision of attention during recognition tests sometimes yields significant effects. Although Baddeley et al. (1984) found no DA effects on recognition accuracy, recognition latencies were significantly lengthened by DA. Beyond this, a pair of studies have shown significant decrements to accuracy when attention is divided during retrieval (Fernandes \& Moscovitch, 2000; Hicks \& Marsh, 2000). ${ }^{1}$

Fernandes and Moscovitch (2000) had participants engage in two simultaneous memory retrieval tasks. The recognition test was a continuous test in which a series of words were presented every 1 sec on the computer screen. The participants had to manually indicate on the computer keyboard whether each word had been previously presented. In the DA condition, the participants simultaneously recalled words from an earlier study list. Performance on recognition test was greatly decreased while the participants were simultaneously engaged in recall (in comparison with a baseline condition in which recognition was the sole task). Although the results indicate a DA deficit in recognition, Fernandes and Moscovitch (2000) argued that decrements obtained were not due to competition for central attentional resources but, rather, were due to competition for word-specific representational systems (i.e., both the recall and the recognition tests involved verbal material). Although Fernandes and Moscovitch (2000) theorized that retrieval is not necessarily obligatory, as Craik et al. (1996) claimed, their conclusion that memory retrieval is not affected by competition for central resources is consistent with the view of Craik et al. (see Fernandes \& Moscovitch, 2002, 2003, for similar conclusions).

Hicks and Marsh (2000), in contrast, posited that competition for central resources can lead to a DA effect under certain conditions. They made this claim by appealing to the dual-process model of recognition memory, theorizing that encoding conditions producing high levels of recollection would lead to such competition. Before describing the details of Hicks and Marsh's study, we need first to describe the main ideas of the dual-process model of recognition memory.

Dual-process models propose that recognition memory consists of two independent processes, recollection and familiarity (see Yonelinas, 2002, for a review). Recollection is viewed as consciously remembering both the specific test item and the context in which it occurred, whereas familiarity is described as an undifferentiated feeling that an item was previously encountered, without a retrieval of contextual details. In this model, recollection is assumed to involve a recall-like search process that is consciously controlled and intentional. Familiarity, however, is assumed to be unconscious and unintentional, reflecting processing fluency. Because the recollection process is assumed to be effortful, slower, and intentional, it is assumed to require attentional resources, whereas familiarity does not (Yonelinas, 2002).

Several methods have been used to tease apart these two processes, such as process dissociation and remember/ know procedures (Gardiner \& Richardson-Klavehn, 2000; Jacoby, 1991). Studies in which these procedures have been used have reported that various manipulations produce differential effects on the purported measures of recollection and familiarity, with such dissociations helping to further delineate the natures of the dual processes (Yonelinas, 2002). To take one prominent example, semantic (i.e., deep) encoding greatly enhances measures of recollection but has much less effect on familiarity (Gardiner, 1988; Gardiner, Java, \& Richardson-Klavehn, 1996).

For present purposes, the most important distinction between recollection and familiarity is that the former is purported to be attention demanding, whereas the latter is assumed to be automatic. In support of this view, applications of the process dissociation procedure imply that dividing attention at retrieval reduces recollection (e.g., Jacoby, 1991; Mulligan \& Hirshman, 1997). Thus, in contrast to Craik et al. (1996; see also Naveh-Benjamin et al., 1998; Naveh-Benjamin, Craik, Gavrilescu, \& Anderson, 2000; Naveh-Benjamin, Craik, Perretta, \& Tonev, 2000), the dual-process model implies that certain aspects of recognition memory retrieval are sensitive to the dividing of attention during retrieval.

Appealing to the dual-process model, Hicks and Marsh (2000) argued that prior studies of DA and retrieval used encoding conditions that failed to elicit later attentiondemanding recollection. In previous studies, participants simply read or heard a list of words. Later, memory for the words was tested, using a standard recognition test under either FA or DA conditions. Hicks and Marsh argued that simply reading words is a relatively shallow encoding task, unlikely to produce recollective retrieval. Memory performance in these studies would, therefore, be unaffected by dividing attention (Hicks \& Marsh, 2000). Rather, Hicks and Marsh argued, deeper encoding conditions, those that enhance conceptual or distinctive processing, are necessary for inducing later recollection and susceptibility to the effects of dividing attention.

To this end, Hicks and Marsh (2000) manipulated the likelihood of recollection by having participants encode items either by reading the words in their intact form or by generating words presented as anagrams (i.e., the participants had to switch two nonadjacent transposed letters). Hicks and Marsh argued that the anagram condition 
induces deeper encoding, leading to more recollection during the subsequent recognition test and, thus, more susceptibility to divided attention. The intact words, in contrast, should be encoded relatively shallowly, producing little recollection and rendering these test items less susceptible to DA effects. As a preliminary result, Hicks and Marsh found that under full attention, words encoded as anagrams were better remembered than items encoded intact, showing a typical generation effect (e.g., Slamecka $\&$ Graf, 1978). More important, words encoded as anagrams were more affected by dividing attention than were words encoded intact.

Hicks and Marsh (2000) explained their results in terms of the dual-process model, arguing that recollection-based retrieval, like that produced by deep encoding conditions, produces a reliance on attention during retrieval. Although Hicks and Marsh's account focused predominantly on the attentional demands of recollective retrieval, their results and analysis also imply a hypothesis about the type of encoding that leads to attention-demanding retrieval. Specifically, research on dual-process models of recognition uniformly indicates that deep, semantic levels of encoding produce greater recollection than do shallower, non-semantic-encoding conditions. Indeed, deep or conceptual encoding is one of the most robust ways to produce recollection-based retrieval (for a review, see Yonelinas, 2002). Consistent with these results, Hicks and Marsh referred to recollective, attention-demanding retrieval as that produced by deeper encoding conditions, whereas non-attention-demanding (familiarity-based) retrieval is a product of shallower encoding conditions. Before continuing, we have one comment about terminology. In the levels-of-processing (LoP) tradition (e.g., Craik \& Lockhart, 1972; Craik \& Tulving, 1975), the deep encoding condition typically requires semantic processing of the study item, whereas the shallow condition focuses on nonsemantic attributes. In keeping with this tradition, we will use the terms deep and semantic encoding interchangeably.

This discussion indicates two possible implications of Hicks and Marsh's (2000) analysis. The first is that deep, or semantic, encoding produces attention-demanding retrieval (via enhanced recollection). We will refer to this as the semantic-encoding hypothesis. The second is that recollection, regardless of how it is induced, produces retrieval susceptible to DA. Hicks and Marsh emphasized the second of these notions. In the present study, we extended Hicks and Marsh's analysis by investigating the first.

It should be noted that Hicks and Marsh's (2000) experiments were not designed to directly assess the semanticencoding hypothesis, and although their results are consistent with this hypothesis, several questions remain. First, the "deeper" encoding conditions in Hicks and Marsh's study consisted of solving simple anagrams (participants simply had to switch two underlined letters). However, recent research indicates that the letter transposition task (along with some other generation tasks) does not produce greater semantic encoding than does reading words intact (Mulligan, 2002).
Mulligan (2002) examined the effects of several generation manipulations on the implicit test of category exemplar production and its explicit counterpart, category-cued recall. Generation tasks that Mulligan labeled nonsemantic (letter transposition and word fragment completion) increased performance for the category-cued recall test but had no effect on category exemplar production. Generation tasks considered semantic (e.g., generating the critical word from a question related to meaning) produced robust generation effects on both category exemplar production and category-cued recall. Given that the category exemplar production test is known to be sensitive to traditional manipulations of semantic encoding (e.g., Mulligan, Guyer, \& Beland, 1999; see Roediger \& McDermott, 1993 , for a review), the results from this study imply that not all generation tasks enhanced this type of encoding. In particular, generating words from nonsemantic cues (such as anagrams or word fragments) does not appear to increase semantic encoding (although it does increase distinctiveness, presumably along a nonsemantic dimension; see Mulligan, 2002, for a discussion). Thus, the letter transposition task used by Hicks and Marsh (2000) may not lead to deeper semantic encoding than does the read condition. This indicates that the semantic-encoding hypothesis has not been fully evaluated. The goal of the first experiment is to assess this hypothesis by using a more traditional LoP manipulation.

Another important issue is whether semantic encoding generally produces attention-demanding retrieval. A first element of generality is whether deep encoding is necessary for attention-demanding retrieval. One way to assess this is to use a recognition test that has little reliance on semantics, such as a perceptually driven (rhyme recognition) test. Second, an alternative account of Hicks and Marsh's (2000) result is that conditions producing high levels of recognition accuracy (regardless of levels of recollection) are affected by DA to a greater degree than are conditions producing lower accuracy. Given that semantic encoding enhances recognition accuracy, it is unclear whether its susceptibility to DA is due to enhanced recollection or higher levels of recognition accuracy. ${ }^{2}$ A second aspect of the generality issue is whether a semantic-encoding condition produces differential reliance on attention even when it does not produce higher overall recognition than does a non-semantic-encoding condition. These two aspects of generality can be assessed in a single experimental design, making use of procedures from research on transfer-appropriate processing, described next.

The traditional LoP framework emphasizes the importance of encoding mechanisms in memory, proposing that deeper, semantic levels of encoding produce optimum memory performance (Craik \& Lockhart, 1972; Craik \& Tulving, 1975). The transfer-appropriate processing (TAP) framework, on the other hand, emphasizes the match between encoding and retrieval processes, arguing that optimal memory performance is achieved when retrieval processes recapitulate encoding (Morris, Bransford, \& Franks, 1977; see also Kolers \& Roediger, 1984; Roediger, 1990). Morris et al. argued that under certain 
circumstances, items encoded in a shallow, nonsemantic manner could be better remembered than more deeply encoded items. In their study, participants encoded items in either a semantic or a rhyme condition. The participants' memory was then tested, using either a standard recognition test or a rhyme recognition test. In the latter test, some of the test words rhyme with study words, and the participant has to recognize which test words rhyme with words presented earlier. The semantically encoded items were better remembered on the standard recognition test, whereas phonetically encoded items were better remembered on the rhyme recognition test (Morris et al., 1977). This demonstrated that semantic encoding does not necessarily produce superior memory; rather, the match between the encoding and the retrieval operations determines the "best" encoding condition. In our study, we will employ a procedure similar to that used in Morris et al.'s study.

The present study had three specific goals. First, we wanted to assess the overall effects of DA on both standard and perceptually based retrieval. Second, we wished to assess the semantic-encoding hypothesis, using an unambiguous manipulation of semantic encoding (i.e., a traditional LoP manipulation). Third, we wished to test the generality of the idea that "deep" encoding produces greater sensitivity to dividing attention during retrieval than does "shallow" encoding.

\section{EXPERIMENT 1}

In Experiment 1, we used an unambiguous LoP manipulation previously shown to enhance semantic encoding (see Morris et al., 1977; Mulligan \& Hirshman, 1997). At study, the participants answered an orienting question that accompanied each study word. Half of the questions were semantic (e.g., Is this a type of tool? DRILL) and half nonsemantic, relating to the word's sound (e.g., Does this word rhyme with spill? DRILL). Furthermore, half of each question type had a correct answer of yes (yes items), and half no (no items). The participants encoded all the items under full attention. The memory test was a standard recognition memory test (as in Hicks \& Marsh, 2000). The participants took the test under either FA or DA. In the FA condition, the participants' sole task was the recognition test. In the DA condition, the participants performed a secondary task while simultaneously taking the recognition test.

There were several expectations about the results. First, we expected better memory for the semantically encoded than for the phonetically encoded items, the traditional LoP effect (e.g., Craik \& Tulving, 1975). Prior research also indicates that question answer affects memory, with yes items producing better recognition than do no items (e.g., Craik \& Tulving, 1975; Mulligan \& Hirshman, 1997). The main issue is the semantic-encoding hypothesis, which predicts that items encoded semantically should be more affected by dividing attention than are phonetically encoded items. Of course, the precondition of this prediction is that there should be at least some effect of DA on recog- nition accuracy. Finally, consideration of the question type variable raises an additional possibility. Craik and Tulving proposed that yes items produce greater integration of the word and the orienting question than do no items. Given that retrieval of the question context provides a basis for an accurate old decision, the yes items are at a relative advantage. This account implies that the advantage for yes items is due to associative (recollective) retrieval, which, in turn, implies that the yes items are more likely to be affected by DA at retrieval than are the no items.

\section{Method}

Participants. Forty-eight undergraduates at the University of North Carolina at Chapel Hill participated in this experiment in exchange for credit in an introductory psychology course.

Design and Materials. We used a 2 (encoding level) $\times 2$ (question type) $\times 2$ (attention at test) $\times 2$ (presentation rate at test) mixed factorial design, with encoding level and question type manipulated within subjects and attention at test and presentation rate manipulated between subjects. There were two levels of encoding, semantic and phonetic. There were two question types, half with a correct answer of yes (e.g., Does this word rhyme with spill? DRILL) and half with a correct answer of no (e.g., Does this word rhyme with flair? DRILL). During the memory test, words were presented at a rate of either one word every $4 \mathrm{sec}$ or one word every $6 \mathrm{sec}$. Two presentation rates were used because a pilot version of Experiment 2 (which used a rhyme recognition test) indicated that a presentation rate of $4 \mathrm{sec}$ was insufficient time and needed to be extended to $6 \mathrm{sec}$. Because we wanted Experiments 1 and 2 to be comparable, half of the participants in Experiment 1 were given a presentation rate of $6 \mathrm{sec}$ in order to match Experiment 2. The other half of the participants received a presentation rate of $4 \mathrm{sec}$ in order to match the typical time used for recognition tests in prior studies (e.g., Anderson et al., 1998).

The study list consisted of 40 five-letter words (mean frequency $=$ 42.08; Kučera \& Francis, 1967). For each word, four orienting questions were constructed. Two of the questions emphasized phonetic aspects of the word, with one question possessing an answer of yes (phonetic-yes; PY) and the other an answer of no (phonetic-no; $\mathrm{PN}$ ). The other two questions emphasized semantic aspects of the word: again, one with a yes answer (semantic-yes; SY) and the other with a no answer (semantic-no; SN). Phonetic questions asked about rhymes, whereas semantic questions asked about either synonymity or category membership. Each word was chosen for the study list so as to meet the following constraints: (1) It bore little semantic or phonetic relation to the other study words, and (2) it rhymed with at least two other (nonstudy) words. In addition, four buffer items were created.

Four versions of the study list were created, counterbalancing items across the question categories (PY, PN, SY, and SN). In each list, there were 10 instances of each question category. The items within each list were randomly ordered, with the following constraints: (1) No more than two instances of a single question category appeared in succession, and (2) each quartile of the list contained an equal number of PY, PN, SY, and SN questions. The recognition test consisted of 40 old items and 40 new items. Like the old items, the new items were five letters, of moderate frequency (mean frequency $=43.78$; Kučera \& Francis, 1967); they were semantically and phonetically unrelated to old and new study items and rhymed with at least one other word.

The secondary task was a working memory task, the $n$-back task. This task was presented visually on the computer screen and consisted of a serial presentation of a string of digits. The participants viewed each digit and made a decision (indicated with a keypress) as to whether it was the same as or different from the preceding digit. The task was self-paced (Craik et al., 1996); the response to one 
digit initiated the display of the next digit. Because the participants compared the current digit with the preceding digit, this task is referred to as the 1-back task. One third of the digits were the same as the preceding digit, and two thirds were different.

Procedure. The participants were tested individually. Prior to the study phase, the participants were informed that they would hear a list of words and that they should try to remember the words for a later memory test (the exact nature of the memory test was not described). The participants were also instructed that prior to each word, a yes/no question would be presented on the computer screen and that they must respond to that question, using the computer keyboard. The participants were then presented with the study list. In each trial, the question appeared on the computer screen $2 \mathrm{sec}$ before the word was presented over the computer speakers. Study words were presented twice in succession, and the participants were informed that they should respond to the question after the second presentation of the word. Pilot testing of the rhyme recognition test used in Experiments 2 and 3 showed that accuracy rates were low with a single presentation. As a result, in all the experiments, the words were presented twice. The question remained on the screen throughout the trial, until the participant pressed the "Y" (yes) or "N" (no) key.

The study phase was followed by a 3-min interval, during which the participants performed the 1-back task by itself. This administration served as a retention interval and as the pretest baseline measure. For this task, the participants viewed a continuous series of digits on the computer screen and made a yes/no judgment about each digit. If the current digit matched the preceding digit, the participants pressed the Y key for yes; if the current digit differed from the preceding digit, the participants pressed the $\mathrm{N}$ key for no. The participants' response determined the rate of presentation; that is, the next digit appeared on the screen immediately after the participants made a response to the current digit. This was designed to mimic the continuous presentation rate of the CRT task previously used to divide attention (e.g., Craik et al., 1996). The participants were told to respond as quickly and as accurately as possible.

The memory test consisted of a standard recognition test. The participants heard a series of words through a stereo. The participants were instructed to listen to each word and say "old" if the test word was also presented during the study list (old items) or say "new" if the test word was not presented during the study list (new items). After hearing the word, half of the participants had $4 \mathrm{sec}$ to make a recognition judgment, whereas the other half had $6 \mathrm{sec}$ to make a decision. The participants took the recognition test under either FA or DA. In the FA condition, the recognition test was the sole task. In the DA condition, the participants performed both the recognition test and the 1-back task. These participants were told that they would take the test while also viewing a string of numbers and that they needed to (1) indicate verbally whether the test word had been presented earlier and (2) simultaneously use the computer keyboard to indicate whether or not the current digit matched the preceding digit. They were told that the recognition test and the 1-back task were equally important and to perform both as accurately as possible.

After the test phase, the participants in the DA condition again performed the 1-back task alone, serving as the posttest baseline measure. The pretest and posttest baselines are used to assess sec- ondary task costs produced by the recognition test. Two baselines are necessary because the secondary task may be subject to practice effects over the course of the experiment. By comparing pretest and posttest baselines, this possibility could be examined.

\section{Results}

Recognition test. Preliminary analyses indicated that presentation time produced no significant effects or interactions. Consequently, we will report all data collapsed over this factor. Table 1 shows the hit and false alarm rates. Recognition accuracy was assessed with corrected hits (hits - false alarms). Analyses based on $d^{\prime}$ accuracy produced the same results. Corrected hit rates (Figure 1) were analyzed with a 2 (encoding level) $\times 2$ (question type) $\times 2$ (attention at test) mixed factorial ANOVA. Unless otherwise specified, all alpha levels were set at .05 . There were four significant effects: a main effect of encoding level $\left[F(1,44)=84.59, M S_{\mathrm{e}}=0.02\right]$, indicating greater accuracy for semantic items than for phonetic items; a main effect of question type $[F(1,44)=23.85$, $\left.M S_{\mathrm{e}}=0.02\right]$, such that yes items were remembered better than no items; a main effect of attention $[F(1,44)=6.36$, $\left.M S_{\mathrm{e}}=0.06\right]$, indicating that dividing attention significantly decreased overall memory performance; and an interaction between question type and attention $[F(1,44)=$ $\left.5.31, M S_{\mathrm{e}}=0.02\right]$, indicating that yes items were affected to a greater degree by dividing attention than were no items. Critically, the interaction between encoding level and attention was nonsignificant $(F<1)$, indicating that semantic and phonetic items were equally affected by dividing attention. All other interactions were also nonsignificant $(F \mathbf{s}<1)$. Because of the interaction of question type and attention, separate analyses were performed on yes items and no items. The effect of DA was significant for yes items $\left[F(1,46)=11.93, M S_{\mathrm{e}}=0.04\right]$, but not for no items $(F<1)$.

Secondary task. Analyses were performed on median RT scores and mean percent correct (accuracy) on the 1-back task. All the participants performed this task during the retention interval (the pretest baseline). However, only the participants in the DA condition completed the posttest baseline and DA conditions. Preliminary analyses compared the pretest baseline performance in the FA and DA conditions and showed no significant difference in either RT $($ FA mean $=503.00$; DA mean $=554.23)$ or accuracy $($ FA mean $=.95$; DA mean $=.96)$.

The secondary task results for the DA group are presented in Table 2 . These results were analyzed with a series of $t$ tests. First, for the RT data, the participants

Table 1

Hit Rates and False Alarm Rates for the Standard Recognition Test in Experiment 1

\begin{tabular}{|c|c|c|c|c|c|}
\hline & \multirow{3}{*}{$\begin{array}{c}\text { Question } \\
\text { Type }\end{array}$} & \multicolumn{4}{|c|}{ Encoding Level } \\
\hline & & \multicolumn{2}{|c|}{ Full Attention } & \multicolumn{2}{|c|}{ Divided Attention } \\
\hline & & Phonetic & Semantic & Phonetic & Semantic \\
\hline \multirow[t]{2}{*}{ Hits } & Yes & .72 & .90 & .66 & .85 \\
\hline & No & .55 & .75 & .62 & .77 \\
\hline False alarms & & \multicolumn{2}{|c|}{.11} & \multicolumn{2}{|c|}{.19} \\
\hline
\end{tabular}




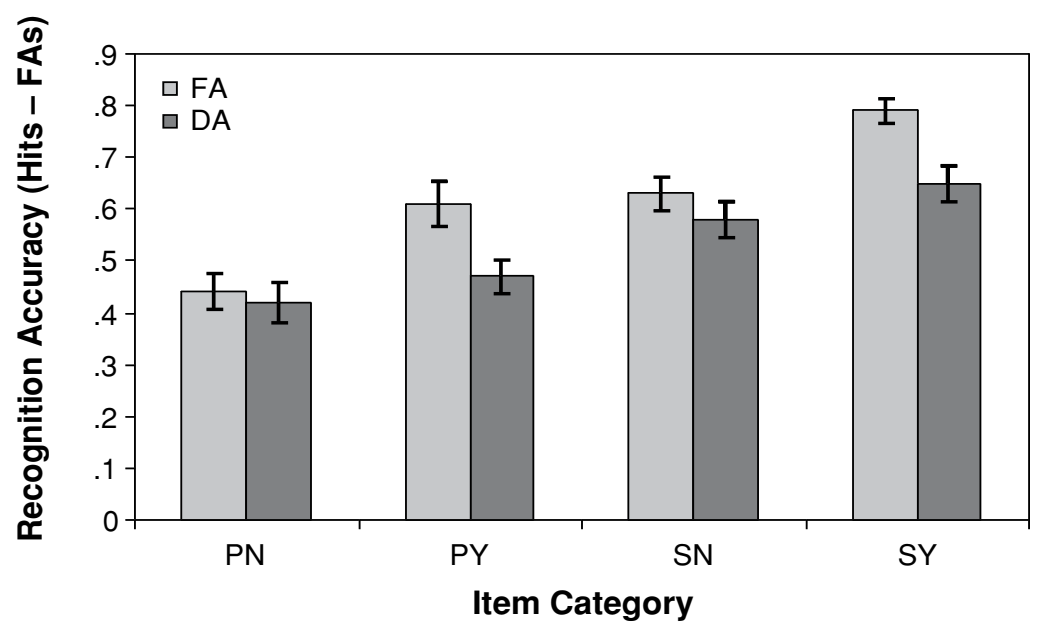

Figure 1. Mean accuracy (hits - false alarms [FAs]) on the standard recognition test as a function of item category type. PN, phonetic-no; PY, phonetic-yes; SN, semantic-no; SY, semantic-yes; FA, full attention; DA, divided attention. Error bars represent \pm 1 standard error.

were significantly faster in the posttest than in the pretest condition $[t(23)=5.66]$, indicating that the 1-back task exhibited practice effects. There was no difference in preand posttest accuracy $[t(23)=0.49]$, presumably because pretest accuracy was already near ceiling. DA secondary task performance was analyzed relative to the average of the pre- and posttest baseline conditions. There was a significant increase in RTs when the 1-back task was performed in conjunction with the memory test $[t(23)=$ 4.93]. There was also a significant decrease in accuracy $[t(23)=5.43]$. The same pattern of results was obtained when the DA condition was compared with the pretest baseline condition $(p \mathrm{~s}<.05)$, which provides a more conservative assessment of secondary task costs.

\section{Discussion}

Several aspects of the results merit comment, beginning with recognition performance. First, as was expected, semantic encoding produced better memory than did phonetic encoding (Craik \& Tulving, 1975). Second, yes items were better remembered than no items, also replicating earlier research. Third, dividing attention during memory retrieval did have an overall effect on memory accuracy, replicating some prior studies (Hicks \& Marsh, 2000; Mulligan \& Hirshman, 1997) but not others (Craik et al., 1996; Naveh-Benjamin et al., 1998). This result joins other results in arguing against the idea that retrieval in recognition is obligatory or protected (Fernandes \& Moscovitch, 2000, 2002, 2003; Hicks \& Marsh, 2000).

More critically, attention and encoding condition did not interact; contrary to the semantic-encoding hypothesis, the negative effect of DA was not larger for semantic than for phonetic encoding. The present results provide no evidence that semantic encoding per se induces later attention-demanding retrieval. More generally, these results bear on Hicks and Marsh's (2000) primary focus, that recollective retrieval (no matter how induced) is suscep- tible to DA effects. Because semantic encoding produces more recollection than do shallower encoding conditions (Yonelinas, 2002), this view leads to the same expectation as the semantic-encoding hypothesis in the present case, that the semantic condition should be affected more by DA than is the phonetic condition. Such was not found.

Interestingly, whereas semantic and phonetic items were equally affected by dividing attention, yes items were more affected by dividing attention than were no items. As was noted in the introduction, this is consistent with the notion that yes questions produce greater integration of the word with its question context and that retrieval of the question context might be differentially harmed by DA. If the question word integration is thought of as a general type of elaborative encoding, rather than as a more specific semantic encoding (Craik \& Tulving, 1975), this might point to an extension of the semantic-encoding hypothesis. That is, elaborative encoding in general, rather than semantic encoding in particular, might induce attention-demanding retrieval (via recollection). We will return to this point in greater detail in the General Discussion section.

It should be noted that during the study portion of the experiment, items were presented twice in succession. This was part of our attempt to make Experiment 1 as comparable as possible to the succeeding experiments (see the Method section for details). However, there is some evidence that massed repetition enhances both recollec-

Table 2

Mean of Median Reaction Time (RT, in Milliseconds) and

Percent Correct (Accuracy) for Each Condition of the 1-Back Task in Experiment 1 for the DA Group

\begin{tabular}{lcc}
\hline \multicolumn{1}{c}{ Condition } & RT & Accuracy \\
\hline Pretest baseline & 554.23 & 96.05 \\
Posttest baseline & 487.98 & 96.00 \\
With memory test & 607.98 & 91.04 \\
\hline
\end{tabular}


tion and familiarity (e.g., Jacoby, 1999; Jacoby, Jones, \& Dolan, 1998; Parkin, Gardiner, \& Rosser, 1995; Parkin \& Russo, 1993; Rajaram, 1993; Yonelinas, 2002; cf. Dewhurst \& Anderson, 1999). This prompted one reviewer to wonder whether the repetition might have imbued the shallowly encoded items with additional recollection, mitigating differences in recollection between deep and shallow items. This seems unlikely to be an important determinant of our pattern of results. First, both deep and shallow items were presented twice. If repetition enhanced recollection, it should have done so for both conditions, leaving a large recollection advantage for the deep items (consistent with the large LoP effect observed). Second, if repetition-induced recollection played a meaningful role, the no items would have been subject to its effects and might be expected to exhibit a DA effect. This was not observed.

Another interesting aspect of these data comes from the comparison of the PY and SN conditions. One concern raised in the introduction is that susceptibility to DA effects might be driven by overall accuracy levels, with conditions producing higher levels of accuracy exhibiting greater sensitivity to DA. Contrary to this concern, the PY condition is significantly affected by DA $[t(46)=2.63]$, whereas the SN condition is not $(|t|<1)$, even though the $\mathrm{SN}$ condition produces slightly higher levels of recognition accuracy under FA. This argues against a simple scaling artifact in explaining the effects of DA on recognition memory.

Finally, the results from the secondary task analyses replicate prior research (Craik et al., 1996; Naveh-Benjamin et al., 1998), demonstrating that secondary task performance is disrupted by simultaneous memory retrieval.

\section{EXPERIMENT 2}

Experiment 2 provided a further assessment of the semantic-encoding hypothesis, using a perceptually driven rhyme recognition test. The experiment assessed whether phonetically encoded items, semantically encoded items, or both are affected by DA on a perceptual test of memory. To this end, the participants encoded items in the same manner as in Experiment 1. However, at test, the participants were given a rhyme recognition test in which they had to remember whether the test word rhymed with a word presented during the study phase. The typical LoP effect is reversed on the rhyme recognition test: On this test, phonetically encoded words produce greater accuracy (Morris et al., 1977). Experiment 2 also provided the first test of the effects of DA on a perceptually driven memory test.

Attention was divided during the test phase only, with half of the participants tested under FA and the rest under DA. According to TAP, in the FA condition, recognition accuracy should be higher for the items encoded phonetically, replicating prior results (Morris et al., 1977). With regard to attention, the semantic-encoding hypothesis argues that semantically encoded items are more susceptible to DA effects. In contrast, if DA effects are driven by performance level, the phonetic condition should be more susceptible to DA effects.

\section{Method}

Participants. Seventy-three undergraduates at the University of North Carolina at Chapel Hill participated in this experiment in exchange for credit in an introductory psychology course.

Design, Materials, and Procedure. The design, materials, and procedure were the same as those in Experiment 1, with two exceptions. First, Experiment 2 used the rhyme recognition of Morris et al. (1977), in which the participants indicated whether the test word rhymed with a word that was heard in the study list. The old items in the rhyme recognition test rhymed with one, and only one, word in the study list. If the question category for the study word was PY, the rhyme word in the test was not the same as the rhyme word in the study question. The new items on the rhyme test were the same as those used in Experiment 1. New foils on the test did not rhyme with any of the words presented during the study phase. However, each foil had at least one other rhyme, so that it could not be excluded on that basis. As in the standard recognition test, the rhyme test consisted of 40 old items and 40 new foils, which were presented aurally. Second, only a single presentation time (of $6 \mathrm{sec}$ ) was used.

\section{Results}

One participant was eliminated due to technical problems, leaving an effective sample size of $n=72$.

Rhyme recognition test. Table 3 shows the hit rates and false alarm rates. The corrected hit rates (Figure 2) were analyzed using a 2 (encoding level) $\times 2$ (question type) $\times 2$ (attention at test) mixed factorial ANOVA. There was a main effect of encoding level $[F(1,70)=$ $\left.5.70, M S_{\mathrm{e}}=0.02\right]$, indicating that phonetically encoded items were remembered better than semantically encoded items. The main effect of question type was also significant $\left[F(1,70)=21.95, M S_{\mathrm{e}}=0.02\right]$, so that yes items were remembered better than $n o$ items. Finally, there was a significant main effect of attention at test $[F(1,70)=5.16$, $\left.M S_{\mathrm{e}}=0.08\right]$, indicating that dividing attention significantly reduced recognition accuracy. The only interaction that reached significance was that between encoding level and question type $\left[F(1,70)=18.70, M S_{\mathrm{e}}=0.02\right]$, indicating that the phonetic-semantic difference was greater for yes items than for no items. It is important to note that the interaction between encoding level and attention at test was nonsignificant $(F<1)$, indicating a comparable DA effect for semantically and phonetically encoded items. All other interactions were also nonsignificant $\left(F_{\mathrm{s}}<1\right)$.

Secondary task. Analyses were performed on both median RT and percent correct for each participant. As in Experiment 1, pretest performance between the FA group and the DA groups did not differ on either RT (FA mean = 529.81 ; DA mean $=511.26)$ or accuracy $($ FA mean $=.95$; DA mean $=.95)$. For the DA group, both the RT and the accuracy data (Table 4) were analyzed with a series of $t$ tests. First, RTs were significantly shorter in the posttest than in the pretest condition $[t(35)=4.58]$, indicating practice effects. However, there were no differences in accuracy, presumably because accuracies were near ceiling $(|t|<1)$. As a result, DA secondary task costs were assessed relative to the average of the pre- and posttest baseline conditions. Replicating the results from Experi- 
Table 3

\begin{tabular}{lccccccc}
\multicolumn{8}{c}{ Hit Rates and False Alarm Rates for the } \\
Rhyme Recognition Test in Experiment 2 \\
\hline
\end{tabular}

ment 1 , there were significant DA effects for the RT data $[t(35)=6.75]$ and for the accuracy data $[t(35)=6.64]$. A similar pattern of results was found when the DA condition was compared with the pretest baseline condition.

\section{Discussion}

The results of the FA condition demonstrate that our materials and procedures were effective in replicating the results in Morris et al. (1977) in detail. First, phonetic encoding led to better memory than did semantic encoding. Thus, the traditional LoP effect was reversed with a perceptual memory test. Second, yes items were better remembered than no items. Third, the reversed LoP effect occurred for the yes items but not for the no items.

Dividing attention significantly decreased memory accuracy. This argues against the hypothesis that memory retrieval is an obligatory process in this perceptually based recognition test. In addition, phonetically and semantically encoded items were affected to an equivalent degree by dividing attention. As in Experiment 1, we found no support for the semantic-encoding hypothesis. That is, enhanced semantic encoding did not lead to a greater reliance on attention during retrieval. It is worth noting that there was a nonsignificant trend in the opposite direction: Phonetically encoded items were numerically (but not sig- nificantly) more affected by dividing attention than were semantically encoded items.

Although it has previously been reported that dividing attention during retrieval can diminish memory performance, no previous study has used a perceptual (or data-driven) memory test to examine these effects. This experiment indicates that even when retrieval relies on perceptual (nonsemantic) information, dividing attention still significantly reduced performance. This indicates that semantic processing is not necessary for DA effects in retrieval, because the rhyme test has little reliance on semantic information. If semantic processing generally produces attention-demanding retrieval, one would expect that either (1) the semantic condition would be more affected by DA or (2) a perceptually driven memory test would be relatively unaffected by DA. Neither outcome occurred, arguing that semantic processing does not generally induce attention-demanding retrieval.

The findings for secondary task costs extend much prior research (Craik et al., 1996; Naveh-Benjamin et al., 1998) demonstrating that secondary task performance is impaired by simultaneous memory retrieval. Even with a perceptually driven memory test, significant secondary task costs were found. This and the memory test data indicate that perceptually driven memory retrieval is generally

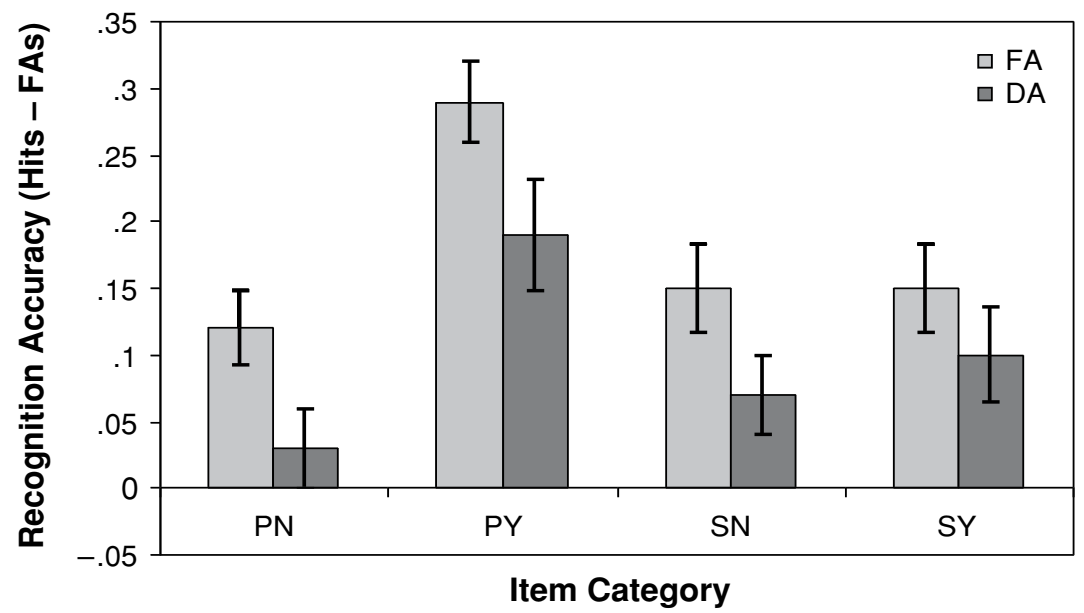

Figure 2. Mean accuracy (hits - false alarms [FAs]) on the rhyme recognition test as a function of item category type. PN, phonetic-no; PY, phonetic-yes; SN, semantic-no; SY, semantic-yes; FA, full attention; DA, divided attention. Error bars represent \pm 1 standard error. 
Table 4

Mean of Median Reaction Time (RT, in Milliseconds) and Percent Correct (Accuracy) for Each Condition of the 1-Back Task in Experiment 2 for the DA Group

\begin{tabular}{lcc}
\hline \multicolumn{1}{c}{ Condition } & RT & Accuracy \\
\hline Pretest baseline & 511.26 & 94.76 \\
Posttest baseline & 455.60 & 94.12 \\
With memory test & 573.71 & 88.21 \\
\hline
\end{tabular}

an attention-demanding process, as is retrieval on conceptually driven tests.

\section{EXPERIMENT 3}

The nonsignificant trend toward the phonetic items being more affected by DA warrants further investigation. It is possible that the materials used in Experiment 2 may have limited our abilities to observe an interaction between encoding level and attention. The number of items in each of the four experimental categories was comparatively small (i.e., only 10 words in each category). In addition, the use of orienting questions to induce semantic and phonetic encoding necessitated that half of the questions had yes answers, whereas the other half had no answers. To the extent that yes and no items differ from each other, the number of items in each condition is diluted. For example, in Experiment 2, only the yes items produced the reversed LoP effect. Thus, only half of the phonetic items (i.e., the PY items) contributed to this critical effect. Experiment 3 addressed this issue by reframing each question as a semantic or a phonetic generation task (e.g., $A$ word that rhymes with spill. DR__), thus eliminating the factor of question type. This eliminates potential differences between yes and no items, and doubles (to 20) the number of items in each of the two encoding conditions.

\section{Method}

Participants. Thirty-two undergraduates at the University of North Carolina at Chapel Hill participated in this experiment in exchange for credit in an introductory psychology course.

Design and Materials. We used a 2 (encoding level) $\times 2$ (attention at test) mixed factorial design, with encoding level manipulated within subjects and attention at test manipulated between subjects. The materials were identical to those in Experiment 2, with the following modification. The orienting questions were transformed into a series of statements and word stems. Word stems consisted of the first two or three letters of each word, followed by a blank. The statements were clues about the identity of the word stem and were either semantic (e.g., A type of tool: DR__, for DRILL) or phonetic (e.g., A word that rhymes with spill: DR__, for DRILL). This manipulation yielded two encoding conditions (semantic and phonetic), with 20 items in each category.

Procedure. During the study phase, the participants were presented visually with a series of statements and word stems. The participants were instructed to use the statement as a clue about the identity of the word and to generate a word that began with letters shown on the screen. The participants said the word aloud. After $8 \mathrm{sec}$, regardless of whether or not the word was properly generated, the word was presented aurally through the computer speakers. After the encoding phase, the participants were given a retention task for $3 \mathrm{~min}$, followed by a rhyme recognition test, both identical to those used in Experiment 2.

\section{Results}

Rhyme recognition test. Table 5 shows the hit and false alarm rates. The corrected hit rates (Figure 3) were analyzed using a 2 (encoding level) $\times 2$ (attention at test) mixed factorial ANOVA. There was a main effect of encoding level $\left[F(1,30)=16.87, M S_{\mathrm{e}}=0.01\right]$, indicating that phonetic items were remembered with greater accuracy than were semantic items; an effect of attention $\left[F(1,30)=4.18, M S_{\mathrm{e}}=0.04\right]$, indicating that DA significantly reduced memory accuracy; but no interaction between encoding level and attention $(F<1)$, indicating a comparable DA effect for semantically and phonetically encoded items.

Secondary task. As in earlier experiments, pretest baseline secondary task performance was compared for participants in the FA group versus the DA group. The groups did not differ significantly on RT (FA mean $=$ 535.03; DA mean $=505.50)$ or accuracy (FA mean $=$ .96 ; DA mean $=.95)$. The data for each condition are presented in Table 6. RTs were significantly faster in the posttest than in the pretest condition $[t(15)=3.23]$, but there were no differences in accuracy $(|t|<1)$. DA secondary task costs were assessed relative to the average of the pre- and posttest baseline conditions. Replicating the results of Experiment 2, there were significant DA effects for the RT data $[t(15)=5.64]$ and for the accuracy data $[t(15)=-8.03]$. A similar pattern of results were found when the DA condition was compared with the pretest baseline condition only, a more conservative test of secondary task costs.

\section{Discussion}

The results replicate those of Experiment 2 and from Morris et al. (1977) in demonstrating a reversal of the LoP effect. That is, phonetically encoded items were remembered better than semantically encoded items. This indicates that our modified materials produced the same fundamental effect as the original materials. In addition, dividing attention at test significantly reduced memory performance on the rhyme recognition test, as in Experiment 2 . These results provide further evidence that retrieval is attention demanding for this perceptually driven test.

Most critically, there was no interaction between attention and encoding level. Even when all the study items were concentrated into the conditions that exhibit the reversed LoP effect, semantic and phonetic items were affected similarly by dividing attention. This implies that the failure to observe such an interaction in Experiment 2 was

Table 5

Hit Rates and False Alarm Rates for the Rhyme Recognition Test in Experiment 3 Encoding Level

\begin{tabular}{|c|c|c|c|c|}
\hline & \multicolumn{4}{|c|}{ 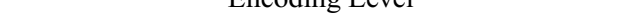 } \\
\hline & \multicolumn{2}{|c|}{ Full Attention } & \multicolumn{2}{|c|}{ Divided Attention } \\
\hline & Phonetic & Semantic & Phonetic & Semantic \\
\hline Hits & .59 & .49 & .53 & .44 \\
\hline False alarms & \multicolumn{2}{|c|}{.23} & \multicolumn{2}{|c|}{.27} \\
\hline
\end{tabular}




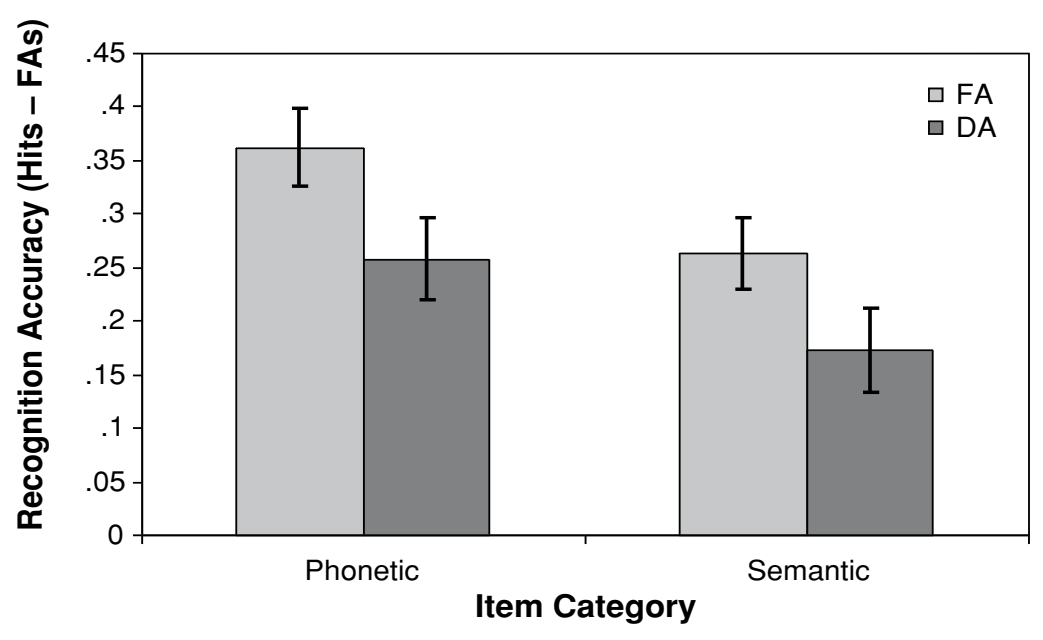

Figure 3. Mean accuracy (hits - false alarms [FAs]) on the rhyme recognition test as a function of item category type. FA, full attention; DA, divided attention. Error bars represent \pm 1 standard error.

not produced by the relatively low number of items in the effective (PY and SY) encoding conditions. It appears that DA affects the two encoding conditions equally (indeed, the reductions in accuracy produced by DA were nearly identical: .10 for phonetic and .09 for semantic).

Furthermore, the pattern of results from the secondary task replicates those from Experiments 1 and 2 and much prior research (Craik et al., 1996; Naveh-Benjamin et al., 1998). As in Experiment 2, the secondary task data and the memory test data indicate that perceptually driven memory retrieval is generally an attention-demanding process.

\section{GENERAL DISCUSSION}

Memory retrieval has been characterized as automatic or obligatory by some researchers (Baddeley et al., 1984; Craik et al., 1996; Naveh-Benjamin et al., 1998), as susceptible to material-specific interference by others (e.g., Fernandes \& Moscovitch, 2000), and as reliant on central attentional resources by still others (e.g., Hicks \& Marsh, 2000; Jacoby, 1991). The present study extends the investigation of attention and memory retrieval. First, we wished to assess the effects of dividing attention during retrieval on standard and perceptually based retrieval. Second and more critically, we assessed the semantic-encoding hypothesis in two ways. First, we compared the effects of DA at retrieval within semantic- and non-semanticencoding conditions, using an unambiguous manipulation of semantic encoding. Second, we examined whether semantic or phonetic items would be affected by DA on a test relatively insensitive to semantic encoding, the rhyme recognition test.

We first will consider the results of the standard recognition test (Experiment 1). Overall, recognition accuracy was affected by dividing attention, conflicting with some results (e.g., Baddeley et al., 1984; Craik et al., 1996) but consistent with others (Hicks \& Marsh, 2000). Why the discrepancy? The semantic-encoding hypothesis proposes that, via recollection, deep encoding is necessary to elicit attention-demanding retrieval and that earlier research (which typically failed to show a DA effect) was limited to shallow encoding conditions unlikely to elicit recollective retrieval. This account does not accommodate the present results, because attention produced equivalent effects on semantic and phonetic items.

An alternative is suggested by the observation that DA effects were larger for yes than for no items on the standard recognition test. As has been noted by Craik and Tulving (1975), differences between yes and no items are unlikely due to semantic encoding: "It does not seem intuitively reasonable that words associated with yes responses require deeper processing before the decision is made. However, if high levels of retention are associated with 'rich' or 'elaborate' encodings of the words (rather than deep encoding), the differences in retention between positive and negative words become understandable" (p. 281; italics in original). Under this view, the yes questions facilitate the integration of the studied word and its encoding (question) context into a coherent memory trace. Such integration can assist recognition accuracy. During the test, a yes item might lead to a retrieval of the question, which would be positive evidence of "oldness." No items, however, are less likely to lead to question retrieval (Craik \& Tulving, 1975; Morris et al., 1977). As a result, the richer encoding context leads to a greater overall memory.

Table 6

Mean of Median Reaction Time (RT, in Milliseconds) and Percent Correct (Accuracy) for Each Condition of the 1-Back Task in Experiment 3 for the DA Group

\begin{tabular}{ccc}
\multicolumn{4}{c}{ Task in Experiment 3 for the DA Group } \\
\hline Condition & RT & Accuracy \\
\hline Pretest baseline & 505.50 & 94.73 \\
Posttest baseline & 458.59 & 94.38 \\
With memory test & 555.47 & 87.00 \\
\hline
\end{tabular}


Such retrieval of contextual information is typically conceived of as recollection (e.g., Yonelinas, 2002). Consequently, the present results and analysis are an extension of Hicks and Marsh's (2000) general claim that conditions promoting recollection produce attention-dependent retrieval. In addition, the results of Experiment 1 imply an important disparity with the semantic-encoding hypothesis: It is not semantic encoding per se but, rather, elaborative encoding that leads to attention-demanding retrieval on a standard recognition test.

This provides a basis for comparison with prior research. Specifically, the no items of Experiment 1 were associated with a nonsignificant drop in accuracy of .035. This is quite similar to the nonsignificant drops found in prior studies (e.g., Anderson et al., 1998; Baddeley et al., 1984; Craik et al., 1996). Yes items, however, were associated with a significant drop of .135. To the extent that prior studies (Anderson et al., 1998; Baddeley et al., 1984; Craik et al., 1996) did not induce elaborative encoding (analogous to no items in the present experiments), one might expect the null effects of DA on a standard recognition test (cf. Hicks \& Marsh, 2000).

The results of Experiments 2 and 3, using the perceptually driven rhyme recognition test, provide additional evidence regarding the semantic-encoding hypothesis. First, the rhyme recognition test is relatively insensitive to semantic encoding (as evidenced by the reversed LoP effect). Given that test accuracy was significantly reduced by DA, it appears that semantic information is not necessary for attention-demanding retrieval. Convergent is the finding that the semantic-encoding condition produced no greater reliance on attention than did phonetic encoding. Indeed, in none of the present experiments were semantically encoded items affected more by DA than were phonetically encoded items.

Why, then, was the rhyme recognition test affected by DA? One possibility follows the recollection account described above. According to this view, if a condition leads to recollective recognition (e.g., retrieval of contextual details from the study episode), that condition will be susceptible to DA. It seems likely that the rhyme recognition test differs from standard recognition in just this way; this test generally requires the participant to retrieve the studied word, given the rhyming word as a cue, regardless of the encoding condition. If retrieving contextual details is considered a form of attention-demanding recollection, retrieving the central information of the event would also be a form of attention-demanding recollection.

This may help explain why the effects of attention were moderated by question type for standard recognition (Experiment 1), but not for rhyme recognition (Experiment 2). In the case of standard recognition, retrieval of question context is not necessary for recognition, but it may be helpful when this information is available (i.e., for the yes items). Thus, recollection is more likely for yes than for no items, with differential susceptibility to DA the result. In the case of rhyme recognition, recollecting the study word may be the primary basis for recognition (i.e., undifferentiated familiarity may not be as useful or as heavily used as in standard recognition). If so, this form of recollection would be dominant for all conditions, rendering them equally susceptible to the negative effects of DA. In this sense, the rhyme recognition test might be more akin to cued recall than to standard recognition. Given that DA effects are typically greater for cued recall than for standard recognition (e.g., Craik et al., 1996), the overall DA effects found in Experiments 2 and 3 may be consistent with these prior results.

More generally, it is significant that DA affected rhyme recognition at all. Earlier research on memory retrieval used standard recognition and recall tests (e.g., Baddeley et al., 1984; Craik et al., 1996; Hicks \& Marsh, 2000; Iidaka, Anderson, Kapur, Cabeza, \& Craik, 2000; Mulligan \& Hirshman, 1997), tests typically conceived of as conceptually driven (e.g., Jacoby, 1983; Roediger, 1990). To date, no study has assessed the effects of dividing attention on a perceptual, data-driven memory test. The present results indicate that perceptually driven retrieval can be just as affected by dividing attention as retrieval that is conceptually driven. In sum, the present results do not support the notion that retrieval on either rhyme recognition or standard recognition is automatic or obligatory.

Next, we will return to the study by Hicks and Marsh (2000) that initially motivated these studies. We have argued that Hicks and Marsh's "deep" encoding condition (a letter transposition, generate task) may not actually enhance semantic encoding, relative to a reading condition (Mulligan, 2002). This possibility motivated our use of a traditional LoP manipulation. Our results indicate that semantic encoding per se did not produce attentiondemanding retrieval. The account that we have sketched out above, focusing on recollection of specific study information (study item, question context, etc.), adequately accounts for our results. Applied to the results in Hicks and Marsh, this account argues that the letter transposition condition produced greater sensitivity to DA because that condition produced greater study elaboration and, ultimately, greater reliance on recollection. It does, however, argue that deep or semantic encoding is not a necessary condition in producing attention-demanding retrieval. Rather, it could be the case that the letter transposition condition produced better encoding of study context. For instance, in comparison with the reading condition, the letter transposition condition might produce superior encoding (and later recollection) of the cognitive operations used to encode the word (e.g., Johnson, Hashtroudi, \& Lindsay, 1993). Alternatively, other aspects of the study context might be better encoded in the letter transposition condition (although see Mulligan, 2004). In either case, the greater recollection (and susceptibility to DA effects) need not be mediated by semantic information, although this idea certainly requires additional inquiry.

The present results help to extend our understanding of when distractor tasks induce DA effects on a memory test. Fernandes and Moscovitch $(2000,2002,2003)$ found that the similarity between the memory retrieval test and the secondary task induces material-specific interference (e.g., Fernandes \& Moscovitch, 2000, 2002, 2003). For 
example, if both the memory test and the secondary task require the processing of words, this can cause competition for processing resources for a word-specific representation system and reduce memory performance. However, the present experiments used different material types for the memory test and the secondary task (words in the former, numbers in the latter). Consequently, our results are unlikely to have been due to material-specific interference (the same is true of Hicks \& Marsh, 2000). These DA effects implicate central attentional resources in retrieval for both memory tests (Hicks \& Marsh, 2000). On the basis of the present view (and in line with Hicks and Marsh's view), general-elaborative encoding should produce items with richer memory traces. Remembering such items might include retrieval of other contextual details, involving recollection, which would lead to attentiondemanding retrieval.

Finally, a corollary of the present view is that if recollective retrieval is attention demanding, memory tests that place heavy emphasis on recollection should be similarly attention demanding. Tests for source, context, and order information are typically assumed to rely heavily on recollection, because these tests require the retrieval of specific study episode details (Yonelinas, 2002). Therefore, such tests should be affected by DA during retrieval. Research by Troyer, Craik, and colleagues (Troyer \& Craik, 2000; Troyer, Winocur, Craik, \& Moscovitch, 1999) indicates that this is the case. Similarly, tests of associative recognition should be affected by DA at retrieval. An associative recognition paradigm involves the presentation of a pair of words during initial learning (e.g., A-B, C-D, E-F). During a subsequent memory test, the participants are again presented with word pairs in one of four conditions: (1) intact pairs (i.e., both items in the pair had been studied together; e.g., A-B), (2) rearranged pairs (i.e., both items had been studied but in different pairs; e.g., C-F), (3) old-new pairs (i.e., an old item is presented with a new foil; e.g., D-X), and (4) new pairs (i.e., neither item was presented during study; e.g., Y-Z). On this test, only pairs in the intact condition should be called "old." Because correct associative recognition involves remembering associative details of word pairs, it also meets the typical definition of recollection (Yonelinas, 2002). Castel and Craik (2003) divided attention during encoding and found severe decrements to associative recognition. No study to date, however, has divided attention during retrieval on such a memory test.

\section{REFERENCES}

Anderson, N. D., Craik, F. I. M., \& Naveh-Benjamin, M. (1998). The attentional demands of encoding and retrieval in younger and older adults: I. Evidence from divided attention costs. Psychology \& Aging, 13, 405-423.

Baddeley, A., Lewis, V., Eldridge, M., \& Thomson, N. (1984). Attention and retrieval from long-term memory. Journal of Experimental Psychology: General, 113, 518-540.

Broadbent, D. E. (1958). Perception and communication. London: Pergamon.

Castel, A. D., \& Craik, F. I. M. (2003). The effects of aging and divided attention on memory for item and associative information. Psychology \& Aging, 18, 873-885.
Cherry, E. C. (1953). Some experiments on the recognition of speech with one and two ears. Journal of the Acoustical Society of America, 26, 554-559.

Craik, F. I. M., Govoni, R., Naveh-Benjamin, M., \& Anderson, N. D. (1996). The effects of divided attention on encoding and retrieval processes in human memory. Journal of Experimental Psychology: General, 125, 159-180.

Craik, F. I. M., \& Lockhart, R. S. (1972). Levels of processing: A framework for memory research. Journal of Verbal Learning \& Verbal Behavior, 11, 671-684.

Craik, F. I. M., \& Tulving, E. (1975). Depth of processing and the retention of words in episodic memory. Journal of Experimental Psychology: General, 104, 268-294.

Dewhurst, S. A., \& Anderson, S. J. (1999). Effects of exact and category repetition in true and false recognition memory. Memory \& Cognition, 27, 665-673.

Dodson, C. S., \& Johnson, M. K. (1996). Some problems with the process-dissociation approach to memory. Journal of Experimental Psychology: General, 125, 181-194.

Fernandes, M. A., \& Moscovitch, M. (2000). Divided attention and memory: Evidence of substantial interference effects at retrieval and encoding. Journal of Experimental Psychology: General, 129, 155-176.

Fernandes, M. A., \& Moscovitch, M. (2002). Factors modulating the effect of divided attention during retrieval of words. Memory \& Cognition, 30, 731-744.

Fernandes, M. A., \& Moscovitch, M. (2003). Interference effects from divided attention during retrieval in younger and older adults. Psychology \& Aging, 18, 219-230.

GARDINER, J. M. (1988). Functional aspects of recollective experience. Memory \& Cognition, 16, 309-313.

Gardiner, J. M., Java, R. I., \& Richardson-Klavehn, A. (1996). How level of processing really influences awareness in recognition memory. Canadian Journal of Experimental Psychology, 50, 114-122.

Gardiner, J. M., \& Richardson-Klavehn, A. (2000). Remembering and knowing. In E. Tulving \& F. I. M. Craik (Eds.), Handbook of memory (pp. 229-244). New York: Oxford University Press.

Gruppuso, V., Lindsay, D. S., \& Kelley, C. M. (1997). The processdissociation procedure and similarity: Defining and estimating recollection and familiarity in recognition memory. Journal of Experimental Psychology: Learning, Memory, \& Cognition, 23, 259-278.

Hicks, J. L., \& MARsh, R. L. (2000). Toward specifying the attentional demands of recognition memory. Journal of Experimental Psychology: Learning, Memory, \& Cognition, 26, 1483-1498.

Iidaka, T., Anderson, N. D., Kapur, S., Cabeza, R., \& Craik, F. I. M. (2000). The effect of divided attention on encoding and retrieval in episodic memory revealed by positron emission tomography. Journal of Cognitive Neuroscience, 12, 267-280.

JACOBY, L. L. (1983). Perceptual enhancement: Persistent effects of an experience. Journal of Experimental Psychology: Learning, Memory, \& Cognition, 9, 21-38.

JАСовY, L. L. (1991). A process dissociation framework: Separating automatic from intentional uses of memory. Journal of Memory \& Language, 30, 513-541.

JACOBY, L. L. (1999). Ironic effects of repetition: Measuring age-related differences in memory. Journal of Experimental Psychology: Learning, Memory, \& Cognition, 25, 3-22.

JACOBY, L. L., Jones, T. C., \& Dolan, P. O. (1998). Two effects of repetition: Support for a dual-process model of know judgments and exclusion errors. Psychonomic Bulletin \& Review, 5, 705-709.

JAMES, W. (1890). The principles of psychology. New York: Holt.

Johnson, M. K., Hashtroudi, S., \& LindSAy, D. S. (1993). Source monitoring. Psychological Bulletin, 114, 3-28.

Johnston, W. A., Greenberg, S. N., Fisher, R. P., \& Martin, D. W. (1970). Divided attention: A vehicle for monitoring memory processes. Journal of Experimental Psychology: General, 83, 164-171.

Kolers, P. A., \& Roediger, H. L., III (1984). Procedures of mind. Journal of Verbal Learning \& Verbal Behavior, 23, 425-449.

KuČERA, H., \& FrANCIS, W. N. (1967). Computational analysis of presentday American English. Providence, RI: Brown University Press.

Martin, D. W. (1970). Residual processing capacity during verbal organization in memory. Journal of Verbal Learning \& Verbal Behavior, 9, 391-397. 
Morris, C. D., Bransford, J. D., \& Franks, J. J. (1977). Levels of processing versus transfer appropriate processing. Journal of Verbal Learning \& Verbal Behavior, 16, 519-533.

Mulligan, N. W. (1998). The role of attention during encoding in implicit and explicit memory. Journal of Experimental Psychology: Learning, Memory, \& Cognition, 24, 27-47.

Mulligan, N. W. (2002). The effects of generation on conceptual implicit memory. Journal of Memory \& Language, 47, 327-342.

Mulligan, N. W. (2004). Generation and memory for contextual detail. Journal of Experimental Psychology: Learning, Memory, \& Cognition, 30, 838-855.

Mulligan, N. W., Guyer, P. S., \& Beland, A. (1999). The effects of levels-of-processing and organization on conceptual implicit memory in the category exemplar production test. Memory \& Cognition, 27, 633-647.

Mulligan, N. W., \& Hirshman, E. (1997). Measuring the bases of recognition memory: An investigation of the process-dissociation framework. Journal of Experimental Psychology: Learning, Memory, \& Cognition, 23, 280-304.

MURDOCK, B. B., JR. (1965). Effects of a subsidiary task on short-term memory. British Journal of Psychology, 56, 413-419.

Naveh-Benjamin, M., Craik, F. I. M., Gavrilescu, D., \& Anderson, N. D. (2000). Asymmetry between encoding and retrieval processes: Evidence from divided attention and a calibration analysis. Memory \& Cognition, 28, 965-976.

Naveh-Benjamin, M., Craik, F. I. M., Guez, J., \& Dori, H. (1998). Effects of divided attention on encoding and retrieval processes in human memory: Further support for an asymmetry. Journal of Experimental Psychology: Learning, Memory, \& Cognition, 24, 1091-1104.

Naveh-Benjamin, M., Craik, F. I. M., Perretta, J. G., \& Tonev, S. T. (2000). The effects of divided attention on encoding and retrieval processes: The resiliency of retrieval processes. Quarterly Journal of Experimental Psychology, 53A, 609-625.

Naveh-Benjamin, M., \& Guez, J. (2000). Effects of divided attention on encoding and retrieval processes: Assessment of attentional costs and a componential analysis. Journal of Experimental Psychology: Learning, Memory, \& Cognition, 26, 1461-1482.

Norman, D. A. (1969). Memory and attention: An introduction to human information processing. New York: Wiley.

Park, D. C., Smith, A. D., Dudley, W. N., \& Lafronza, V. N. (1989). Effects of age and a divided attention task presented during encoding and retrieval on memory. Journal of Experimental Psychology: Learning, Memory, \& Cognition, 15, 1185-1191.

Parkin, A. J., Gardiner, J. M., \& Rosser, R. (1995). Functional aspects of recollective experience in face recognition. Consciousness \& Cognition, 4, 387-398.

Parkin, A. J., \& Russo, R. (1993). On the origin of functional differences in recollective experience. Memory, 1, 231-237.

RAJARAM, S. (1993). Remembering and knowing: Two means of access to the personal past. Memory \& Cognition, 21, 89-102.

Roediger, H. L., III (1990). Implicit memory: Retention without remembering. American Psychologist, 45, 1043-1056.

Roediger, H. L., III, \& MCDermott, K. B. (1993). Implicit memory in normal human subjects. In F. Boller \& J. Grafman (Eds.), Handbook of neuropsychology (pp. 63-131). Amsterdam: Elsevier.

Slamecka, N. J., \& Graf, P. (1978). The generation effect: Delinea- tion of a phenomenon. Journal of Experimental Psychology: Human Learning \& Memory, 4, 592-604.

Troyer, A. K., \& Craik, F. I. M. (2000). The effect of divided attention on memory for items and their context. Canadian Journal of Experimental Psychology, 54, 161-171.

Troyer, A. K., Winocur, G., Craik, F. I. M., \& Moscovitch, M. (1999). Source memory and divided attention: Reciprocal costs to primary and secondary tasks. Neuropsychology, 13, 467-474.

Trumbo, D., \& Milone, F. (1971). Primary task performance as a function of encoding, retention, and recall in a secondary task. Journal of Experimental Psychology, 91, 273-279.

Yonelinas, A. P. (2002). The nature of recollection and familiarity: A review of 30 years of research. Journal of Memory \& Language, 46, 441-517.

\section{NOTES}

1. Interestingly, four other studies using the process dissociation (PD) procedure (Jacoby, 1991) have shown decrements to recognition memory when attention was divided during retrieval. In this paradigm, participants encode items from two different study lists (e.g., lists of words heard aloud, read intact, or solved as anagrams) and are then tested on old items from both lists and new items never previously encountered. The participants are then given two types of test instructions, referred to as the inclusion and exclusion conditions. Instructions for the inclusion condition inform the participants to call an item old no matter from which list it came. In the exclusion condition, the participants are told to call an item old only if it originated in the second list. Not all of the questions addressed by the studies are relevant for present purposes. However, the inclusion condition is similar to a standard recognition test. In each of the four studies in which the PD procedure was used, DA reduced accuracy (Dodson \& Johnson, 1996; Gruppuso, Lindsay, \& Kelley, 1997; Jacoby, 1991; Mulligan \& Hirshman, 1997).

2. Hicks and Marsh (2000) attempted to test this alternative by including an additional encoding condition that would increase accuracy without increasing recollection. To this end, they included a condition in which participants read words twice in succession. It was found that accuracy for the seen-twice condition was comparable to that for the anagram condition. However, words in the anagram condition were affected more by DA than were those in the seen-twice condition. Even with this experiment, the results of Hicks and Marsh's study do not completely discredit this alternative. A closer look at Hicks and Marsh's Experiment 4 shows that in the FA test condition, recognition accuracy $\left(d^{\prime}\right)$ for the seen-twice and anagram conditions was quite similar (1.74 vs. 1.89 , respectively), and both were significantly greater than that for the seenonce condition (1.18). When attention was divided during retrieval, recognition accuracy declined to 0.73 (a decrease of 0.45 ) in the seen-once condition, to 1.01 (a drop of 0.73 ) in the seen-twice condition, and to 0.98 (a decline of 0.91 ) in the anagram condition. Although the decline in accuracy for words in the anagram condition was found to be larger than the decline for the seen-twice words, it is apparent that the numerical rank order of FA accuracy is identical to the numerical rank order of susceptibility to DA.

(Manuscript received February 11, 2005; revision accepted for publication June 14, 2005.) 